\title{
Análisis Del Reconocimiento Hacia Los Estudiantes Y Su Influencia En La Producción Académica Universitaria
}

\author{
María José Mirón-Chacón, (MSC) \\ Antonieta Donaji Becerra-Ferniza, (MF) \\ Silvia Sosol-Sánchez, (MAE) \\ Rosalba Segura-Nolasco, (MPG) \\ Mario Páez-Bulbarela, (LC)
}

Colegio Interdisciplinario de Especialización, Orizaba, Veracruz, México

Instituto Tecnológico Superior de Huatusco

Manuel González-Pérez, (PhD)

Colegio Interdisciplinario de Especialización, Orizaba, Veracruz, México

Sistema Nacional de Investigadores (nivel 1)

URL:http://dx.doi.org/10.19044/esj.2020.v16n1p50

\section{Resumen}

La Ley de Educación Superior del Ecuador (LOES) manifiesta que uno de los fines de la educación superior es la producción científica y a las transferencias e innovaciones tecnológicas como un aporte al desarrollo del pensamiento universal (Asamblea Nacional, 2018). La presente investigación tiene como objetivo analizar el reconocimiento hacia los estudiantes que participan en la investigación y su influencia en la producción académica universitaria. Se desarrolló un cuestionario con 23 preguntas sobre los factores que influyen en la producción académica, con un nivel de confianza de1 $95 \%$. El instrumento se aplicó a una muestra de 25 estudiantes del Instituto Tecnológico Superior de Huatusco. Con los datos obtenidos se aplicó una prueba de contraste para validar las hipótesis. Los resultados obtenidos en la Chi observada (8.695652174) y Chi crítica (3.841458821), la hipótesis nula se rechaza, y por lo tanto, las variables son dependientes. Esto confirma la hipótesis de investigación "Si participo en un grupo de investigación entonces soy más valorado por la institución". Se percibe que el estudiante está consciente de que su participación en la investigación científica es reconocida por la comunidad académica. Además de beneficia al reconocimiento de las IES e impacta a los indicadores de producción científica. 
Palabras clave: Reconocimiento, motivación, producción académica universitaria

\title{
Analysis of Recognition Towards Students and Their Influence on University Academic Production
}

\author{
María José Mirón-Chacón, (MSC) \\ Antonieta Donaji Becerra-Ferniza, (MF) \\ Silvia Sosol-Sánchez, (MAE) \\ Rosalba Segura-Nolasco, (MPG) \\ Mario Páez-Bulbarela, (LC)
}

Colegio Interdisciplinario de Especialización, Orizaba, Veracruz, México

Instituto Tecnológico Superior de Huatusco

Manuel González-Pérez, (PhD)

Colegio Interdisciplinario de Especialización, Orizaba, Veracruz, México

Sistema Nacional de Investigadores (nivel 1)

\begin{abstract}
The Law of Higher Education of Ecuador (LOES) states that one of the aims of higher education is scientific production and technological transfers and innovations as a contribution to the development of universal thinking (Asamblea Nacional, 2018). The purpose of this research is to analyze the recognition towards the students who participate in the research and their influence on the academic production. A questionnaire was developed with 23 questions about the factors that influence academic production, with a 95\% confidence level. The instrument was applied to a sample of 25 students of the Higher Technological Institute of Huatusco. With the data obtained, a contrast test was applied to validate the hypotheses. The results obtained in the Chi observed (8.695652174) and Critical Chi (3.841458821), the null hypothesis is rejected, and therefore, the variables are dependent. This confirms the research hypothesis "If I participate in a research group then I am more valued by the institution." It is perceived that the student is aware that their participation in scientific research is recognized by the academic community. It also benefits the recognition of HEIs and impacts the indicators of scientific production.
\end{abstract}


Keywords: Recognition, motivation, academic university production

\section{Introduction}

Los estudiantes deben dominar las competencias para trabajar en equipos multidisciplinarios cuando desarrollen un proceso de investigación.

El estudiante adquiere durante su formación las siguientes competencias (Contreras et al., 2018):

a) Pensamiento crítico

b) Espíritu emprendedor

c) Creatividad

d) Autogestión

e) Experiencia del trabajo en equipo

Existen estudios relacionados a los factores que influyen en la adquisición de competencias para trabajo en equipo en Instituciones de Educación Superior (IES). Por ejemplo, la Ley de Educación Superior del Ecuador (LOES) manifiesta, que uno de los fines de la educación superior es la producción científica (Asamblea Nacional, 2018).

Bastidas et al.(2016) investigaron sobre los factores que motivan los docentes hacia la producción científica en la Universidad Politécnica Salesiana del Ecuador. Sin embargo, estos investigadores no han indagado sobre la motivación de los estudiantes hacia la investigación.

Por otra parte, conviene analizar la necesidad institucional por publicar. Para las IES pertenecientes al Tecnológico Nacional de México (TecNM), es requisito cumplir con evaluaciones departamentales cada seis meses (Dirección de Docencia e Innovación Educativa, 2018). Se evalúa a los docentes en los siguientes ámbitos (Tecnológico Nacional de México, 2015):

a) Docencia

b) Tutoría

c) Vinculación

d) Gestión

e) Investigación

No obstante, la actividad investigativa de los estudiantes no es reconocida en los instrumentos de evaluación. Como resultado de esta actividad no reconocida, los docentes que asesoran a los egresados en sus trabajos de titulación, no los estimulan para la publicación de los resultados (Mayta-Tristán, 2016); aun cuando esto incide en los niveles e indicadores de la productividad académica en las IES. 
Considerando éstos y otros antecedentes, la presente investigación tiene como objetivo analizar el reconocimiento hacia los estudiantes que participan en la investigación y su influencia en la producción académica universitaria.

\section{Metodología}

Para la presente investigación, se consideró a la población del área académica del Instituto Tecnológico Superior de Huatusco (ITSH), una Institución perteneciente al TecNM de la zona centro de Veracruz, donde se consideró a la población del área académica del ITSH (Tabla 1).

Tabla 1 Población del ITSH por estratos.

\begin{tabular}{|c|c|}
\hline Estratos & Población \\
\hline Personal Administrativo & 11 \\
\hline Personal Docente & 72 \\
\hline Alumnos & 2382 \\
\hline Total & 2465 \\
\hline
\end{tabular}

Fuente de Consulta: Elaboración propia, 2019.

La población del alumnado del ITSH, mostrada en la Tabla 2, se clasifica en tres categorías: el sistema escolarizado que asiste de lunes a viernes a clase, el sistema sabatino (también llamado flexible) al que acuden estudiantes que comúnmente trabajan entre semana, y la extensión Coscomatepec, quienes asisten los sábados en otra ciudad donde ofrece sus servicios del ITSH.

Tabla 2 Población por sistema del ITSH.

\begin{tabular}{|l|c|c|c|c|}
\hline Criterio & Escolarizado & Sabatino & $\begin{array}{c}\text { Extensión } \\
\text { Coscomatepec }\end{array}$ & Total \\
\hline Cantidad de alumnos & 876 & 1128 & 378 & 2382 \\
\hline $\begin{array}{l}\text { Porcentaje respecto al } \\
\text { total de la población }\end{array}$ & $37 \%$ & $47 \%$ & $16 \%$ & $100 \%$ \\
\hline
\end{tabular}

Fuente de Consulta: Elaboración propia, 2019.

Como parte del estudio, se desarrolló un cuestionario (Drew et al., 2007) para entrevistar a 25 estudiantes del ITSH, pertenecientes al sistema escolarizado y seleccionados por muestreo determinístico (Rodríguez, 2012) debido a la disponibilidad institucional para realizar la encuesta. Al cuestionario consistente en 23 preguntas sobre los factores que influyen en la producción académica, se validó mediante correlación de Pearson (Díaz et al., 2001). La variable independiente es el reconocimiento profesional y la variable dependiente es el interés personal por desarrollar artículos científicos. La investigación incluye las gráficas de distribución y de ojiva de Chi cuadrada de las variables. Se contrastó y graficó la función inversa teórica del 
p valor contra Chi cuadrada de 1 grado de libertad en la dicotómica (González, 2016).

Además de la encuesta aplicada, se realizó una entrevista a profundidad (Taylor et al., 2008) determinística con un estudiante del ITSH de octavo semestre, identificado en la institución como un alumno activo en proyectos de investigación, promoción y difusión de su carrera y la institución, con dos artículos publicados en revista arbitrada e indexada. En la entrevista, respecto al tema de la importancia del reconocimiento de los estudiantes y su influencia en la producción académica universitaria, se preguntó lo siguiente:

1. ¿En algún momento ha sentido que sus opiniones son más valoradas que sus compañeros, debido a su trayectoria académica?

2. ¿Considera que tiene mayor reconocimiento profesional debido a su participación en investigaciones académicas?

3. ¿Considera que la Institución da promoción al trabajo académico de los alumnos?

4. ¿Considera que su participación en eventos académicos e investigación le facilitó obtener una beca como residente?

Desarrollar la entrevista a profundidad, se hizo con el fin de contrastar las respuestas y formas de pensar de los estudiantes con las respuestas obtenidas de una entrevista a grupos focales o de enfoque (Kitzinger et al., 2004) del área académica de la IES-T, realizada previamente a la entrevista a profundidad.

Los instrumentos de recolección de datos cualitativos fueron validados mediante la revisión expertos investigadores del sistema tecnológico y dos universidades extranjeras.

\section{Resultados y discusión}

La encuesta se desarrolló aplicando el cuestionario a los estudiantes, se validó por correlación de Pearson. Debido a los resultados obtenidos y la intención de estudiar dos ítems principales:

1. ¿Consideras que los estudiantes que desarrollan actividades de investigación son más valorados en la Institución? (ítem 17).

2. ¿Te gustaría ser parte de un grupo de investigación? (ítem 23).

Se validó mediante prueba de contraste o prueba de independencia de Chi cuadrada (De la Fuente-Fernández, 2016) a los dos ítems del estudio para correlacionarlos. Lo anterior, se contrastó para generar la hipótesis siguiente: "Si participo en un grupo de investigación entonces soy más valorado por la institución". Con las respuestas de los estudiantes, se procedió a desarrollar los productos lógicos para los ítems (Tabla 3).

Tabla 3 Producto lógico. 


\begin{tabular}{|c|c|c|c|c|c|c|c|c|c|c|}
\hline \multirow{2}{*}{ Encuestado } & \multirow{2}{*}{ Ítem 1 } & \multirow{2}{*}{ Ítem 2 } & \multicolumn{2}{|c|}{ Ítem 1 } & \multicolumn{1}{|c|}{ Ítem 2 } & \multicolumn{5}{c|}{ Producto lógico } \\
\hline & & & SI & NO & SI & NO & sisi & Sino & nosi & nono \\
\hline 1 & 1 & 1 & 0 & 1 & 0 & 1 & 0 & 0 & 0 & 1 \\
\hline 2 & 1 & 2 & 0 & 1 & 1 & 0 & 0 & 0 & 1 & 0 \\
\hline 3 & 1 & 1 & 0 & 1 & 0 & 1 & 0 & 0 & 0 & 1 \\
\hline 4 & 1 & 1 & 0 & 1 & 0 & 1 & 0 & 0 & 0 & 1 \\
\hline 5 & 1 & 1 & 0 & 1 & 0 & 1 & 0 & 0 & 0 & 1 \\
\hline 6 & 1 & 1 & 0 & 1 & 0 & 1 & 0 & 0 & 0 & 1 \\
\hline 7 & 1 & 1 & 0 & 1 & 0 & 1 & 0 & 0 & 0 & 1 \\
\hline 8 & 1 & 1 & 0 & 1 & 0 & 1 & 0 & 0 & 0 & 1 \\
\hline 9 & 1 & 1 & 0 & 1 & 0 & 1 & 0 & 0 & 0 & 1 \\
\hline 10 & 1 & 1 & 0 & 1 & 0 & 1 & 0 & 0 & 0 & 1 \\
\hline 11 & 1 & 1 & 0 & 1 & 0 & 1 & 0 & 0 & 0 & 1 \\
\hline 12 & 2 & 2 & 1 & 0 & 1 & 0 & 1 & 0 & 0 & 0 \\
\hline 13 & 1 & 2 & 0 & 1 & 1 & 0 & 0 & 0 & 1 & 0 \\
\hline 14 & 1 & 2 & 0 & 1 & 1 & 0 & 0 & 0 & 1 & 0 \\
\hline 15 & 2 & 2 & 1 & 0 & 1 & 0 & 1 & 0 & 0 & 0 \\
\hline 16 & 1 & 1 & 0 & 1 & 0 & 1 & 0 & 0 & 0 & 1 \\
\hline 17 & 1 & 1 & 0 & 1 & 0 & 1 & 0 & 0 & 0 & 1 \\
\hline 18 & 1 & 1 & 0 & 1 & 0 & 1 & 0 & 0 & 0 & 1 \\
\hline 19 & 1 & 1 & 0 & 1 & 0 & 1 & 0 & 0 & 0 & 1 \\
\hline 20 & 1 & 1 & 0 & 1 & 0 & 1 & 0 & 0 & 0 & 1 \\
\hline 21 & 1 & 1 & 0 & 1 & 0 & 1 & 0 & 0 & 0 & 1 \\
\hline 22 & 1 & 1 & 0 & 1 & 0 & 1 & 0 & 0 & 0 & 1 \\
\hline 23 & 1 & 1 & 0 & 1 & 0 & 1 & 0 & 0 & 0 & 1 \\
\hline 24 & 1 & 1 & 0 & 1 & 0 & 1 & 0 & 0 & 0 & 1 \\
\hline 25 & 1 & 1 & 0 & 1 & 0 & 1 & 0 & 0 & 0 & 1 \\
\hline & & & $\mathbf{2}$ & $\mathbf{2 3}$ & $\mathbf{5}$ & $\mathbf{2 0}$ & $\mathbf{2}$ & $\mathbf{0}$ & $\mathbf{3}$ & $\mathbf{2 0}$ \\
\hline & & & & $\mathbf{2 5}$ & $\mathbf{2 5}$ & & & $\mathbf{2 5}$ & \\
\hline
\end{tabular}

Fuente de Consulta: Elaboración propia, 2019.

De la Tabla 3, se obtuvieron la Tabla de contingencia (Tabla 4) y la Tabla de valores esperados (Tabla 5):

Tabla 4 Tabla de contingencia

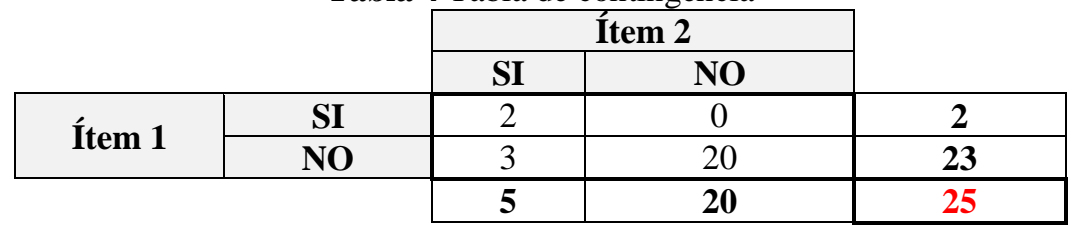

Fuente de Consulta: Elaboración propia, 2019. 
Tabla 5 Tabla de valores esperados

\begin{tabular}{|c|c|c|c|c|}
\cline { 3 - 4 } \multicolumn{2}{c|}{} & \multicolumn{2}{|c|}{ Ítem 2 } \\
\cline { 3 - 5 } \multicolumn{2}{c|}{} & SI & NO & \multicolumn{1}{|c}{} \\
\hline \multirow{2}{*}{ Ítem 1 } & SI & 0.4 & 1.6 & $\mathbf{2}$ \\
\cline { 2 - 5 } & NO & 4.6 & 18.4 & $\mathbf{2 3}$ \\
\hline
\end{tabular}

Fuente de Consulta: Elaboración propia, 2019.

Se obtuvieron los valores para Chi calculada u observada y Chi teórica o crítica, manejando un valor de $95 \%$ de confiabilidad. A continuación en la Tabla 6 se presentan los resultados.

Tabla 6 Tabla de estadísticos Chi cuadrada.

\begin{tabular}{|c|c|c|c|c|c|}
\hline & \multirow{2}{*}{\multicolumn{2}{|c|}{ Ítem 2}} & & \\
\hline & & & & & \\
\hline & & SI & NO & & \\
\hline \multirow{2}{*}{ Ítem 1} & SI & 6.4 & 1.6 & 8 & \\
\hline & NO & 0.556521739 & 0.139130435 & 0.695652174 & \\
\hline & & 6.956521739 & 1.739130435 & 8.695652174 & $\begin{array}{c}\text { Chi calculada } \\
\text { u observada }\end{array}$ \\
\hline & & & & 3.841458821 & $\begin{array}{c}\text { Chi teórica o } \\
\text { crítica }\end{array}$ \\
\hline
\end{tabular}

Fuente de Consulta: Elaboración propia, 2019.

De los resultados obtenidos en la Chi observada que está por arriba de la Chi crítica plasmados en la Tabla 6, la hipótesis nula se rechaza, y por lo tanto, las variables son dependientes. Esto confirma la hipótesis de investigación "Si participo en un grupo de investigación entonces soy más valorado por la institución".

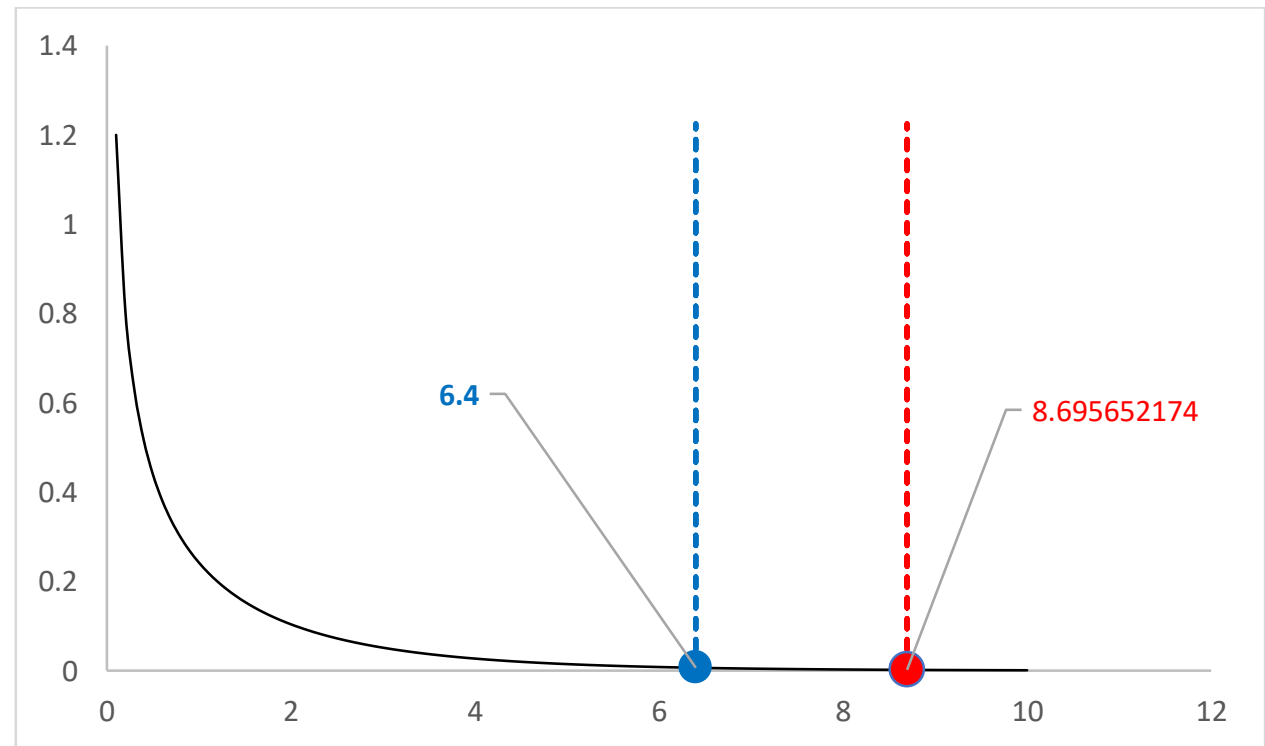


Figura 1 Gráfico de distribución de Chi cuadrada.

Fuente de Consulta: Elaboración propia, 2019.

En la Figura 1 se observa la Chi teórica de color azul y la Chi observada o experimental de color rojo en la gráfica de la distribución de Chi con grado de libertad uno para una dicotómica.

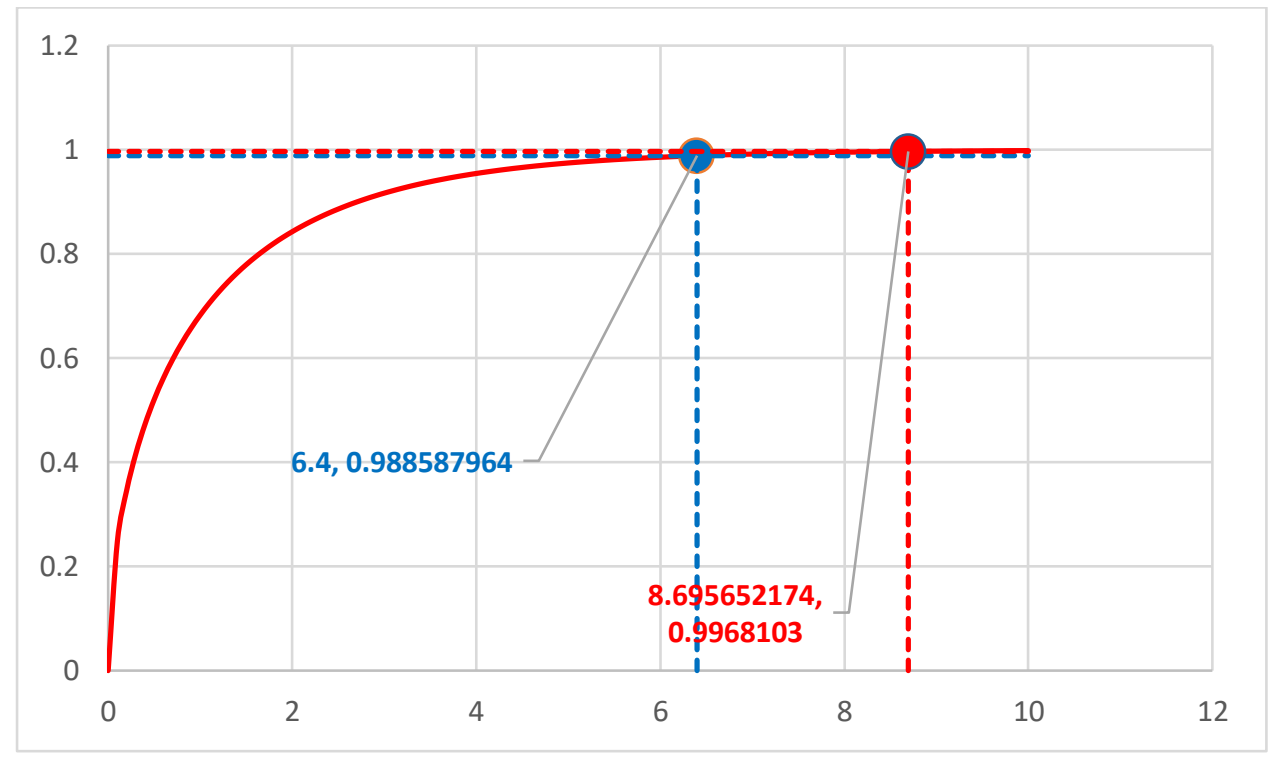

Figura 2 Gráfico de ojiva de Chi cuadrada.

Fuente de Consulta: Elaboración propia, 2019.

En la Figura 2 se observa la Chi teórica de color azul y la Chi observada o experimental de color rojo en la ojiva, se puede notar que la Chi observada tiene un intervalo de confianza del $100 \%$.

Los resultados se reafirman con la entrevista a profundidad realizada, donde se le preguntó a un estudiante seleccionado determinística mente por su trayectoria académica, si en algún momento ha sentido que sus opiniones son más valoradas que sus compañeros, debido a su trayectoria académica. Al respecto, expresó lo siguiente: "Sí. He notado en el salón de clases con diferentes maestros que te ponen un poco más de atención, en este caso a mí como alumno que ha participado en diferentes eventos académicos, como que te ponen un poco más de atención o se concentran más en ti, a revisarte más a detalle todos los trabajos que a otros compañeros, los cuales consideran que no tienen una trayectoria o no dominan el tema, a los cuales los revisan superficialmente a que logren entender la idea del trabajo y que se corrija un poco, pero deberían concentrarse un poco más en las personas que no dominan todos esos temas. En cierta forma te enorgullece que te consideren una persona la cual está preparada para realizar ciertos trabajos y que consideren 
para formar parte de otros (trabajos), y pues que consideren más detalles sobre tu trabajo para que tengan un alcance mayor; pero de cierta forma como te ponen más atención a ti, tienes que dar cada vez un poco más de ti."

El estudiante expresó su opinión acerca de tener mayor reconocimiento profesional debido a su participación en investigaciones académicas. "Espero que sí, porque fue un trabajo el cual en su momento nos costó y se realizaron ciertos pasos para lograr su publicación y que tuviera un alcance. Depende del área en la cual te enfoques, en la investigación se te va a reconocer que a tan temprana edad ya seas coautor en un artículo y capítulo de libro; si te dedicas a otra área quizá no tome demasiada relevancia.”.

Sobre la promoción que la Institución da al trabajo académico de los alumnos, mencionó lo siguiente: "En la Institución existe un departamento académico que se encarga de dar promoción a todas las actividades desarrolladas en el instituto, en su momento fueron estas personas las encargadas de dar vinculación con los diferentes medios (periodísticos) y dar a conocer todos los avances que tuvimos con nuestro proyecto".

Finalmente, se cuestionó al alumno si consideraba que su participación en eventos académicos e investigación le facilitó obtener una beca como residente en una conocida empresa automotriz, a lo que respondió: "Sí. Durante la participación en los eventos académicos vas adquiriendo nuevos conocimientos y mejorando los que ya tenías. Me facilitó mucho el expresarme, antes me costaba mucho el dar a conocer mis ideas ya sea de forma verbal o escrita. Con la participación académica fui mejorando o puliendo todas mis habilidades y he logrado mantenerme destacado entre mis compañeros y así obtener las residencias en el lugar en donde voy a estar."

Confirmando los resultados de la entrevista y encuesta, los resultados se comprueban también por lo expresado por el mismo estudiante y otros de sus compañeros, en la entrevista hacia un grupo focal (Bonilla-Jimenez et al., 2017), realizada como parte del estudio sobre los factores que promueven el trabajo colaborativo y su impacto en la producción académica multidisciplinaria de una Institución de Educación Superior (IES) pública del centro de Veracruz.

\section{Conclusión}

La hipótesis planteada "Si participo en un grupo de investigación entonces soy más valorado por la institución" fue comprobada ampliamente por Chi cuadrada, con un valor de 8.695652174 en Chi observada y 3.841458821 en Chi crítica (Tabla 6, Figura 1).

Se percibe que el estudiante está consciente de que su participación en la investigación científica es reconocida por la comunidad académica. Además de beneficia al reconocimiento de las IES e impacta a los indicadores de producción científica. El factor psicológico es el fundamental partícipe en 
el rendimiento estudiantil, pues un mal estado mental conlleva a un mal estado físico ocasionando un deterioro total del estudiante y un rendimiento bajo (Zambrano et al., 2019).

Se recomienda que docentes y personal académico estimulen a los estudiantes a considerar sus trabajos como material potencialmente publicable y patentable, para lo cual la institución puede considerar la motivación en el puesto de trabajo, las posibilidades de promoción reales, el desarrollo de incentivos a las mejores prácticas docentes, de rendimiento académico como factores que posibilitan un cuerpo docente vocacional y comprometido con la enseñanza y el aprendizaje de su alumnado (Ernesto et al., 2017).

Se agradece ampliamente al ITSH, por la amable disposición de docentes, estudiantes, personal académico y grupos de enfoque (focus group).

\section{References:}

1. Asamblea Nacional (2018). Ley Orgánica de Educación Superior (LOES). Sistema de Información de Tendencias Educativas de América Latina. Organización de las Naciones Unidas para la Educación, la ciencia y la cultura. Recuperado de http://www.siteal.iipe.unesco.org/sites/default/files/sit_accion_files/e c_6011.pdf

2. Bastidas Jimenez, M., \& Benites Medina, R. (2016). Impact of motivation in institutional scientific production. Retos Revista de ciencias de la administración y economía, 6(11), 67-89.

3. Bonilla-Jimenez, F. I., \& Escobar, J. (2017). Grupos focales: una guía conceptual y metodológica.

4. Contreras, J., Rojo, A., Badajós, P., \& Barajas, A. (2018). Desarrollo de competencias para la investigación en Estudiantes de Educación Superior: una experiencia en México. Revista Cognosis, 31(1), 43-50.

5. De la Fuente-Fernández, S. (2016). Aplicaciones de la chi-cuadrado: tablas de contingencia. Homogeneidad. Dependencia e independencia.

6. Díaz, P., \& Fernández, P. (2001). Determinación del tamaño muestral para calcular la significación del coeficiente de correlación lineal. Metodología de la Investigación, 1(6).

7. Dirección de Docencia e Innovación Educativa(2018). Evaluación al desempeño docente de la Educación Superior Tecnológica. Evaluación Departamental. Tecnológico Nacional de México. Recuperado de http://evaldep.ittepic.edu.mx/tecnm/evaldoc/

8. Drew, C. J., Hardman, M. L., \& Hosp, J. L. (2007). Designing and conducting research in education. Sage Publications.

9. Ernesto, T., Torres, J. M. T., \& Artacho, E. G. (2017). Análisis Del Grado De Motivación De Los Profesores En Las Escuelas De 
Enseñanza Primaria De La Periferia De La Zona" B" En Benguela (Angola) En El Desarrollo De Sus Actividades Docentes. European Scientific Journal, 13(14). Recuperado de http://eujournal.org/index.php/esj/article/view/9303/8808

10. González Pérez, Manuel, Juárez Bretón, Silka y Romero Olvera G. Moisés. (2016). Metodología de la redacción Científica. Un enfoque cuántico Hamiltoniano. Columbia S.C. U.S.A.

11. Kitzinger, J., Markova, I., \& Kalampalikis, N. (2004). Qu'est-ce que les focus groups?.

12. Mayta-Tristán, P. (2016). Tesis en formato de artículo científico: oportunidad para incrementar la producción científica universitaria. Acta Médica Peruana, 33(2), 95-98. Recuperado de http://www.scielo.org.pe/pdf/amp/v33n2/a01v33n2.pdf

13. Rodríguez Burgos, K. E. (2012). Investigación cuantitativa: Diseño, técnicas, muestreo y análisis cuantitativo. Metodología para investigaciones de alto impacto en las Ciencias Sociales y Jurídicas (pp. 137-157). Dykinson.

14. Taylor, S. J., \& Bogdan, R. (2008). La entrevista en profundidad. Métodos cuantitativos aplicados, 2, 194.

15. Tecnológico Nacional de México (2015). Instrumento de la evaluación departamental 2015. Registro público del derecho de autor 03-2015-072012524500-01. Secretaría académica, de innovación e investigación. Dirección de docencia e innovación educativa. Recuperado de http://www.dgest.gob.mx/images/areas/docencia01/Libre_para_desca rga/evaluacion_departamental_2015/Instrumento_de_Evaluacion_De partamental_2015_2.pdf

16. Zambrano, G. S. J., Castro, J. E. B., Balseca, M. A., \& Paredes, A. M. (2019). La Migración y su Influencia Académica en los Estudiantes de la Carrera de Ingeniería Mecánica de la Espoch, Ecuador. European Scientific Journal, 15(19). Recuperado de http://eujournal.org/index.php/esj/article/view/12216/11782 